\title{
Confronting the Challenging Asymmetric Addition of Vinyl Arene Pronucleophiles into Ketones: Ligand-Controlled Regiodivergent Processes Through a Dearomatized Allyl-Cu Species
}

\author{
Yuyang Dong ${ }^{\dagger}$, Alexander W. Schuppe ${ }^{\dagger}$, Binh Khanh Mai‡, Peng Liü,*, and Stephen L. Buchwald ${ }^{\dagger}, *$
}

†Department of Chemistry, Massachusetts Institute of Technology, Cambridge, Massachusetts 02139, United States

‡Department of Chemistry, University of Pittsburgh, Pittsburgh, Pennsylvania 15260, United States

\begin{abstract}
The selective reductive coupling of vinyl arenes and ketones represents a versatile approach for the rapid construction of enantiomerically enriched tertiary alcohols. Herein, we demonstrate a CuH-catalyzed regiodivergent coupling of vinyl arenes and ketones, in which the selectivity is controlled by the ancillary ligand. This approach leverages an in situ generated benzyl- or dearomatized allyl-Cu intermediate, yielding either the dearomatized or exocyclic addition products, respectively. The method exhibits excellent regio-, diastereo- and enantioselectivity, and tolerates a range of common functional groups and heterocycles. Computational studies suggest that the regio- and enantioselectivity are controlled by the ancillary ligand, while the diastereoselectivity is enforced by steric interactions between the alkyl-Cu intermediate and ketone substrates in a six-membered cyclic transition state.
\end{abstract}

The enantioselective coupling of two prochiral molecules constitutes an effective strategy for the construction of multiple vicinal stereogenic centers in a single operation. ${ }^{1-}$ 12 In particular, the reaction between prochiral vinyl heterocycles and ketones provides rapid entry to enantiomerically enriched tertiary alcohols, which are prominent substructures in pharmaceuticals and natural products (Figure 1A). ${ }^{12-14}$ Prototypical approaches to form enantioenriched tertiary alcohols have relied on utilizing stoichiometric chiral auxiliaries to relay stereochemical information to the product. ${ }^{15}$ Catalytic asymmetric approaches to access tertiary alcohols generally promote the 1,2-addition of preformed organometallic reagents with a chiral Lewis acid or base catalyst. ${ }^{16-25}$ The application of these protocols, however, is frequently complicated by the pregeneration of a stoichiometric amount of highly reactive carbon nucleophiles, limiting the functional group compatibility. To this end, utilization of olefinic pronucleophiles in conjunction with a catalytic metalhydride represents a versatile approach and obviates the need for preformed organometallic species. ${ }^{7-9,26-33}$

Our group and others have developed asymmetric $\mathrm{CuH}-$ catalyzed carbonyl 1,2-addition reactions utilizing in situ generated allyl-Cu intermediates. ${ }^{34-37}$ This strategy has accommodated a range of olefinic pronucleophiles, ${ }^{38}$ including enynes, ${ }^{12}$ 1,3-dienes, ${ }^{3-4}$ and allenes. ${ }^{5,} 39$ Among these transformations, the allyl-Cu species is proposed to engage the carbonyl substrate through a conserved sixmembered cyclic transition state (I, Figure 1B). ${ }^{3,5}$ However, despite the extensive studies employing these classes of aliphatic olefins, only highly activated vinyl arenes, such as 2-alkenyl azaarenes, have been successfully utilized as pronucleophiles to couple to ketones (Figure 1C). This asymmetric 1,2-addition involves a proposed chair-like transition state in which the $\mathrm{Cu}$-atom is coordinated by the aza-group..$^{40}$ We hypothesized that highly selective coupling between unactivated vinyl aromatic compounds and ketones will likely proceed through the intermediacy of a closed six-membered cyclic transition state, composed of the in situ generated allyl-Cu type intermediate and ketone. ${ }^{41-42}$ A single example of the reductive coupling of a vinyl arene and ketone was reported by our group; however, our attempts to extend this protocol to other substituted styrenes and ketones were unsuccessful (see Scheme S1 in Supporting Information). ${ }^{10,}$ 12, 35, 43-47 The inability to access a six-membered transition state likely forces this reaction to proceed through an unselective fourmembered transition state. We envision that the reduced resonance stabilization of a vinyl heteroarene substrate may produce two equilibrating $\eta^{1}$-benzyl-Cu species (II and III, Figure 1D), in which the position of the $\mathrm{Cu}$ species could be controlled by the steric environment of the ancillary ligand. In the presence of a ketone, an analogous Zimmerman-Traxler-type transition state could be accessed in a regiodivergent fashion (II and III). ${ }^{42,47-65}$ In particular, III provides a dearomative pathway, and the corresponding product allows access to saturated heterocyclic compounds. Accordingly, we describe the ligand-controlled, regiodivergent asymmetric coupling between vinyl heteroarenes and ketones enabled by $\mathrm{CuH}$ catalysts. 
A. Representative pharmaceutical and natual product containing tertiary alcohol

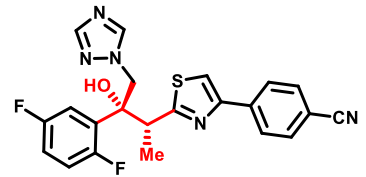

Isavuconazole

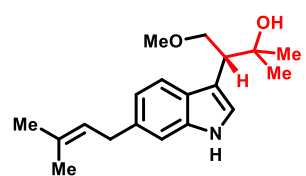

Prenylated Indole Alkaloids
B. CuH-mediated asymmetric reductive coupling of aliphatic olefin pronucleophiles and ketones

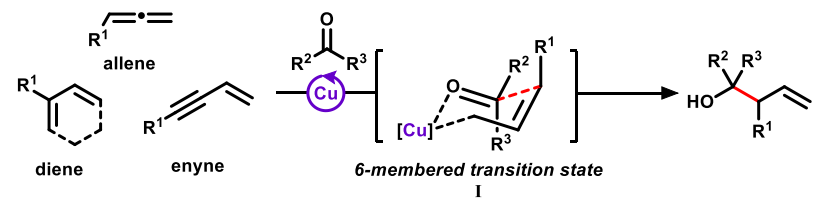

C. CuH-catalyzed addition of alkenyl azaarenes to ketones

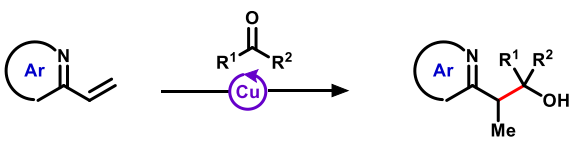

D. Regiodivergent tertiary alcohol synthesis through dearomatized Cu-allyl species (this work)

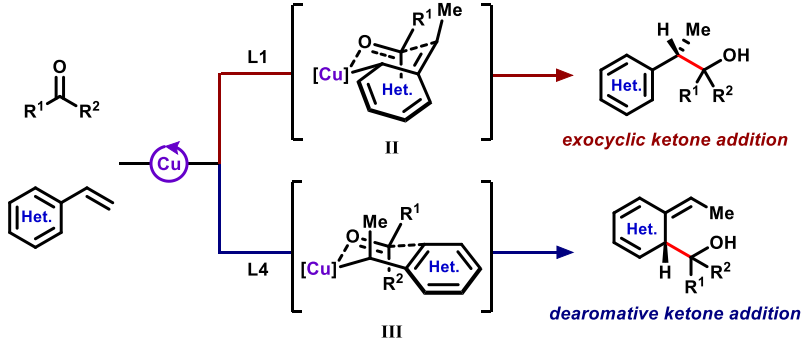

Figure 1. (A) Representative pharmaceutical and natural products containing tertiary alcohol functional groups. (B) $\mathrm{CuH}$-mediated coupling between aliphatic olefin pronucleophiles and ketones through a cyclic, six-membered transition state. (C) Previously reported alkenyl arene to ketone featuring highly activated vinyl azarenes. (D) A catalytic approach to ligand-controlled, regiodivergent coupling between vinyl heteroarenes and ketones using $\mathrm{CuH}$ supported by asymmetric bisphosphines.

We commenced our investigation by examining the reductive coupling of commercially available 3vinylthiophene (1) to acetophenone (2a). Employing several P-stereogenic ligands (L1-L3), ${ }^{66-68}$ the exocyclic product 3a was formed exclusively ( $>20: 1 \mathrm{rr}$ ) in moderate yield and enantioselectivity (entry 1-3, Table 1). We identified the C1-symmetric $(R)-3 \mathrm{H}$-QuinoxP* (L1) as an optimal ligand for this transformation, which provided $\mathbf{3 a}$ with excellent regio-, diastereo-, and enantioselectivity. Lowering the reaction temperature, modifying the copper salt, or reaction solvent were deleterious toward the reaction result (entry 4-6, and Table S1). ${ }^{66-69}$ Decreasing the catalyst loading to $3 \mathrm{~mol} \%$ of $\mathrm{Cu}$ along with $3.6 \mathrm{~mol} \%$ of $\mathbf{L 1}$ was sufficient to achieve full conversion (see SI, Table S1). To inhibit the competing ketone reduction, 2 a could be slowly introduced into the reaction mixture, and the use of an addition rate of $6 \mu \mathrm{L} / \mathrm{min}$ (entry 7) was optimal. While the product yield increased with slower addition, a concomitant decrease in enantioselectivity was observed (see SI, Table S2). Therefore, for operational simplicity, unless otherwise noted, the ketone substrates were added in one portion. To test our aforementioned hypothesis involving a ligand-controlled bifurcation of reactivity, further screening of asymmetric phosphine ligands (see SI, Table S1) revealed the dearomatized product (4a) as a minor regioisomer when chiral bisphosphine $(R, R)-\mathrm{Ph}$-BPE (L4, entry 8) was employed. Therefore, we sought to explore the substrate scope of this ligand-controlled transformation targeting both the exocyclic and dearomative pathways utilizing $\mathbf{L} \mathbf{1}$ and $\mathbf{L 4}$ as the ancillary ligands, respectively.

Table 1. Optimization of the Regio-, Diastereo- and Enantioselective Coupling of 3-Vinylthiophene (1) and Acetophenone (2a) ${ }^{a}$

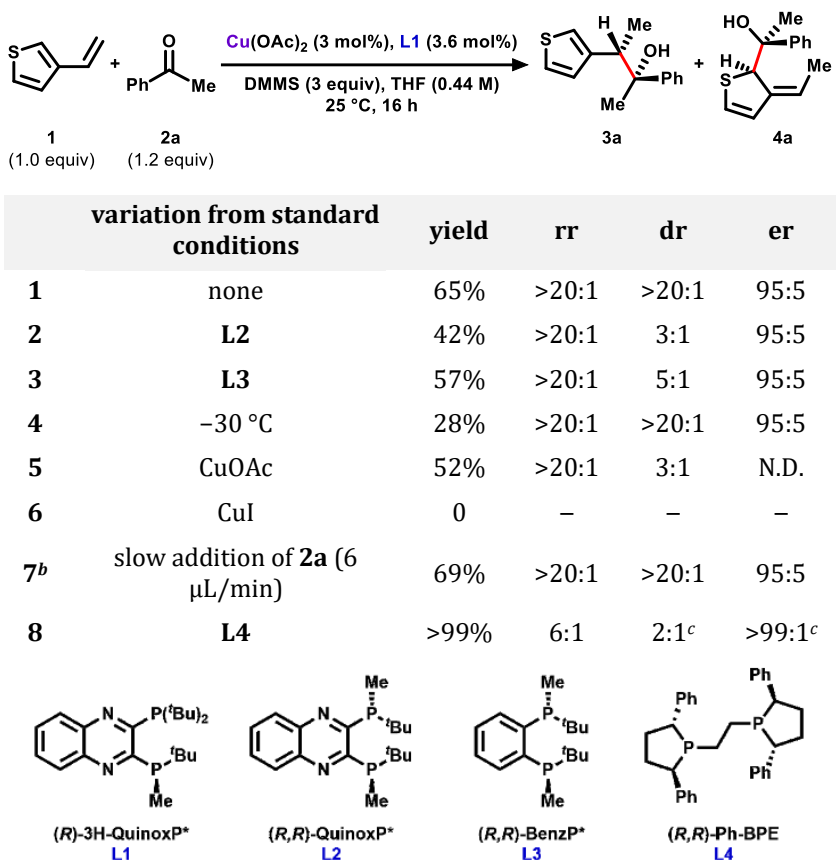

${ }^{a}$ Reaction conditions: $0.20 \mathrm{mmol} 3$-vinylthiophene (1, 1.0 equiv), $0.24 \mathrm{mmol}$ acetophenone ( $2 \mathrm{a}, 1.2$ equiv), and THF ( 0.4 $\mathrm{M})$; reaction yields, regioisomeric and diastereomeric ratios were determined by ${ }^{1} \mathrm{H}$ NMR spectroscopy of the crude reaction mixture using 1,1,2,2-tetrachloroethane as an internal standard (see SI for details). Enantiomeric ratio (er) was determined by chiral supercritical fluid chromatography (SFC). N.D.: not determined. $b \mathbf{2 a}$ was added as a THF solution $(1.2 \mathrm{M})$ to a THF solution of $\mathbf{1}(0.8 \mathrm{M})$ (see SI for experimental details). ${ }^{c}$ Determined for the major regioisomer.

With the optimized reaction conditions identified for the formation of $\mathbf{3 a}$, we assessed the functional group tolerance and scope of compatible heterocycles in the exocyclic addition pathway. The reaction proceeds effectively with substrates bearing both electron-rich and -deficient heterocycles commonly found in pharmaceuticals (Table 2), ${ }^{70-72}$ including a thiophene (3b-f), pyridine (3d), pyrazole (3b), thiazole $(\mathbf{3 g}, \mathbf{3 p})$, pyrrole $(\mathbf{3 j})$, indole $(\mathbf{3 k}-\mathbf{l})$, benzothiophene $(\mathbf{3 l})$, benzofuran $(\mathbf{3 m})$, oxazole $(\mathbf{3 h}-\mathbf{i})$, azaindole (3g, $\mathbf{3 n}-\mathbf{o})$, and carbazole (3h). The relative and absolute configuration of the major enantiomer was determined by obtaining the solid-state structure of two examples (3b, 3i; see SI, Figure S4-5). The products were assigned as the anti-addition product, with the resulting tertiary alcohol as $R$-configuration and $S$ - at the adjacent stereocenter. Acetophenone derivatives featuring ortho(3f), meta- (3j) and para-substituents (3j, 3m-n) are all suitable coupling partners. 
Table 2. Substrate Scope of the Exocyclic Addition of Vinyl Arenes to Ketones ${ }^{a}$

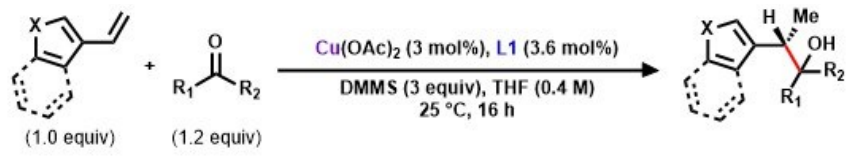

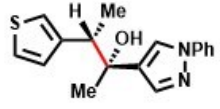

$3 \mathrm{~b}$

$92 \%$ yield

$>20: 1 \mathrm{dr}$

$93.7 \mathrm{er}$

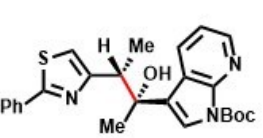

$3 \mathrm{~g}$

$85 \%$ yield

$8: 1 \mathrm{dr}$
$>20: 1 \mathrm{rr}$

$94: 6 \mathrm{er}$

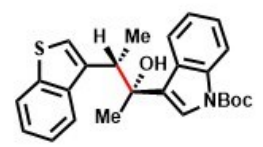

3l
$93 \%$ yield
$>20: 1 \mathrm{dr}$
$>20: 1 \mathrm{rr}$

$>20: 1 \mathrm{rr}$
$94: 6 \mathrm{er}$
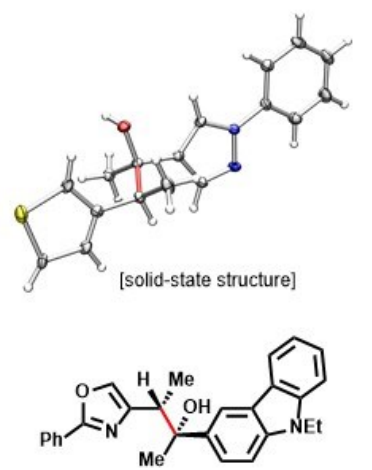

$3 \mathrm{~h}$

$77 \%$ yield
$>20.1 \mathrm{dr}$

$>20: 1 \mathrm{dr}$

>20:1 r

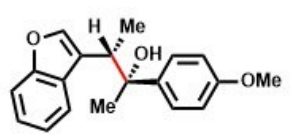

$3 \mathrm{~m}$
$76 \%$ yield
$>20: 1 \mathrm{dr}$

$>20: 1 \mathrm{dr}$
$>20: 1 \mathrm{rr}$

90:10 er
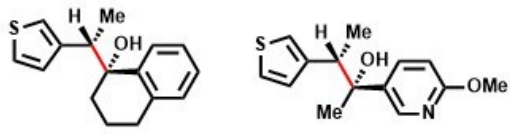

$3 c$

$72 \%$ yield

$11: 1 \mathrm{dr}$
$>20: 1 \mathrm{rr}$

95.5 er

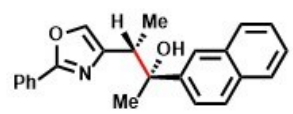

$3 i$

$61 \%$ yield

$>20: 1 \mathrm{dr}$
$>20: 1 \mathrm{rr}$

$>20: 1 \mathrm{rr}$
$93: 7 \mathrm{er}$
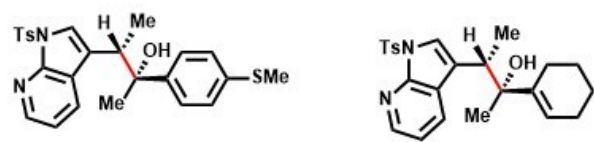

$3 \mathrm{n}$
$75 \%$ yield
$>20: 1 \mathrm{dr}$
$>20: 1 \mathrm{rr}$
$91: 9 \mathrm{er}$
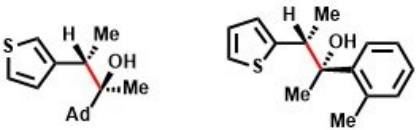

$3 d$
$48 \%$ yield

$16: 1 \mathrm{dr}$

$>20: 1 \mathrm{rr}$
$95.5 \mathrm{er}$

3 e
$33 \%$ yield

$>20: 1 \mathrm{dr}$

$>20: 1 \mathrm{rr}$

$3 f$

$68 \%$ yield

$\begin{array}{ll}1: 1 \mathrm{dr} \\ > & 20: 1 \mathrm{rr}\end{array}$

$91: 9$ er; $85: 15$ er

${ }^{a}$ All yields represent the average of two isolated yields with $0.50 \mathrm{mmol}$ of vinyl heteroarene; regioisomeric (rr) and diastereomeric ratios (dr) were determined by ${ }^{1} \mathrm{H}$ NMR spectroscopy. Enantiomeric ratio (er) was determined by chiral SFC. ${ }^{b} \mathrm{Ketone}$ substrate was added as a THF solution $(2.5 \mathrm{M})$ with $1 \mu \mathrm{L} / \mathrm{min}$ addition rate. ${ }^{c}$ Additional amount of THF $(0.2 \mathrm{M})$ and DMMS (5 equiv) was employed.

Utilization of a cyclic ketone, 1-tetralone, resulted in good enantio- and diastereoselectivity (3c), while an $\alpha, \beta$ unsaturated aliphatic ketone yielded exclusively the 1,2addition product (3o). ${ }^{73-75}$ The reaction conditions also tolerate coordinating groups, such as a thioether $(\mathbf{3 n})$, pyridine-type nitrogen atoms (3b, $\mathbf{3 d}, \mathbf{3 g}-\mathbf{i}, \mathbf{3 n}-\mathbf{p})$, and a tertiary amine (3p) present in antipsychotic haloperidol. A dialkyl ketone was coupled with $\mathbf{1}$ to yield $\mathbf{3 e}$, albeit with lower yield and enantioselectivity.

Intrigued by the significant amount of dearomatized product $\mathbf{4 a}$ observed with $\mathbf{L} \mathbf{4}$ as the ancillary ligand (entry 8 , Table 1 ), we sought to optimize the reaction scope for the dearomative reaction mode. While the coupling between tert-butyl 3-vinyl-1 $H$-indole-1-carboxylate (5) and acetophenone (2a) generated exclusively the exocyclic product with $\mathbf{L} \mathbf{1}$ (cf. Table 2, 3k), utilizing L4 provided the dearomatized product $\mathbf{4 b}$ as the major regioisomer (Table 3). Furthermore, by employing $\mathbf{L} \mathbf{4}$ as the ancillary ligand, a variety of dearomatized heterocycles were obtained in a regioselective manner. A range of heterocyclic substrates, including an indole (4b-c), thiazole (4c), pyridine (4d), azaindole (4e), imidazole (4f), and benzothiophene (4f-g), were well tolerated. A highly reactive 3-oxetanone could be effectively coupled (4d) utilizing this protocol; however, slow addition of the ketone substrate was necessary to minimize the undesired 1,2-reduction pathway. The relative and absolute configuration of the major enantiomer was determined by obtaining the solid-state structure of $\mathbf{4 e}$ (see SI, Figure S6) in conjunction with computational modelling (vide infra). ${ }^{3-4,46}$ The products were assigned as the anti-addition product, with the resulting tertiary alcohol as $R$-configuration. The exocyclic alkene was revealed in the more sterically strained $Z$-configuration, prompting a more detailed analysis of the proposed chair-like transition state by DFT calculations (vide infra). Notably, the generation of dearomatized product features excellent enantiocontrol, > 99:1 er in several cases (4c, 4e-g). To further highlight the synthetic utility of this dearomative transformation, a third stereogenic center could be formed through diastereoselective olefin reduction, targeting highly substituted hydrogenated heterocycles. Hydrogenation of 4g using Pd/C stereospecifically generated the cis-product $\mathbf{4 g}$. When the coupling of vinyl arenes and ketones was conducted on a $5.0 \mathrm{mmol}$ scale, significant heat release was detected. An ice-water bath was utilized to facilitate heat transfer, and preparation of both the exocyclic (3a) and dearomatized products $(\mathbf{4 g})$ were isolated with comparable isolated yield and selectivity on large scale (eq. 1-2).

To explore the origins of the ligand-controlled regiodivergent reaction outcome, density functional theory (DFT) calculations were carried out to study the free energy profiles of the reaction between 5 and 2a featuring both L1 and $\mathbf{L} \mathbf{4}$ as the ancillary ligands. The calculations were performed at the M06/6-311+G(d,p)SDD(Cu)/SMD(THF)//B3LYP-D3/6-31G(d)-

$\operatorname{SDD}(\mathrm{Cu}) / \mathrm{SMD}(\mathrm{THF})$ level of theory (see SI for 
computational details). The hydrocupration of $\mathbf{5}$ with $\mathbf{L 1}$ and L4-supported $\mathrm{Cu}$ catalysts is irreversible (see Figures S8-9), leading to allyl-Cu intermediates $(S)$-Int-1 and $(R)$ Int-1', respectively (Figure $3 \mathrm{~A}$ ). The more energetically accessible hydrocupration transition state is consistent with

Table 3. Substrate Scope of the Dearomative Addition of Vinyl Arenes to Ketones ${ }^{a}$
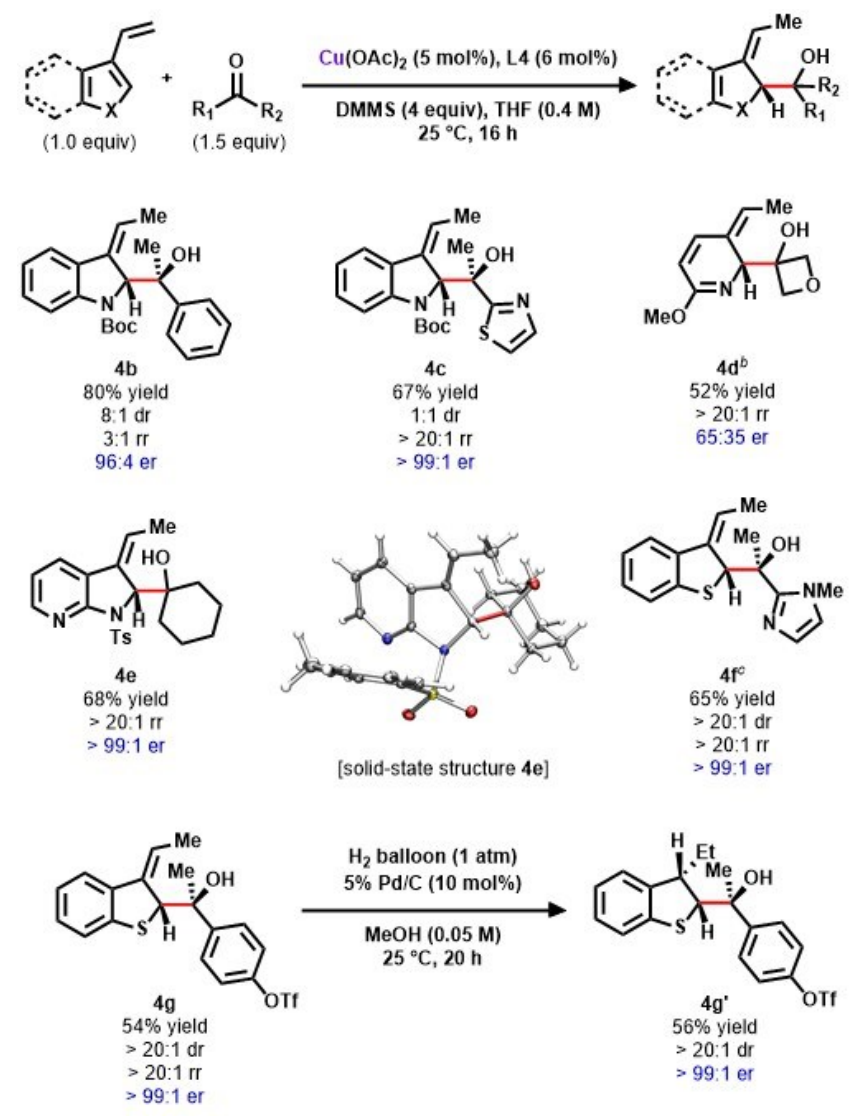

${ }^{a}$ All yields represent the average of two isolated yields with $0.50 \mathrm{mmol}$ of vinyl heteroarenes; regioisomeric ( $\mathrm{rr}$ ) and diastereomeric ratios $(\mathrm{dr})$ were determined by ${ }^{1} \mathrm{H}$ NMR spectroscopy. Enantiomeric ratio (er) was determined by chiral SFC. In order to out compete the ketone reduction, a slightly excess amount of ketone (1.5 equiv) was used. $b 2.0$ equiv of ketone substrate was added as a THF solution (2.2 M) with $3 \mu \mathrm{L} / \mathrm{min}$ addition rate. ${ }^{c}$ Modified amount of ketone $(1.2$ equiv) was employed.
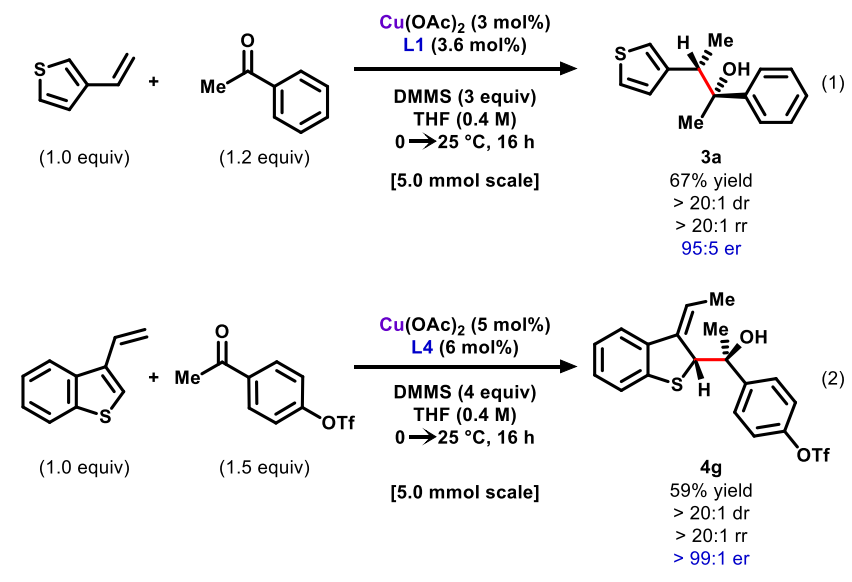

the crystallographically determined absolute configurations of the corresponding carbon atoms $(\mathbf{3 b}, \mathbf{3} \mathbf{i}$ and $\mathbf{4 d}$, vide supra), assuming subsequent stereoretentive 1,3-Cu-migration and ketone trapping steps (vide infra). Alternative hydrocupration pathways are disfavored by placing the heteroarenes in the more sterically encumbered quadrants of the asymmetric ligands.

Due to the facile 1,3-Cu-migration from $(S)$-Int-1 and $(R)$ Int-1' to their respective dearomatized intermediates $(R)$ Int-2 and (S)-Int-2' (Figure 3A), both the regio- and diastereoselectivity of the transformation are under CurtinHammett control. Therefore, the experimentally observed regioisomeric and diastereomeric ratios are determined by the relative energies of the Zimmerman-Traxler-type ketone addition transition states. Our calculations revealed a $5.1 \mathrm{kcal} / \mathrm{mol}$ preference toward the generation of $3 \mathbf{k}$ (TS2) using $\mathbf{L 1}$ and a $1.7 \mathrm{kcal} / \mathrm{mol}$ kinetic barrier difference favoring the production of dearomatized $4 \mathbf{b}$ (TS-3') employing L4, in excellent agreement with the regioselectivity observed in both cases experimentally (>20:1 rr for L1; 3:1 rr for L4). The divergent regioselectivity is controlled by matching of the chair-like activated complex and the chiral pockets created by the ligands as well as the stabilizing $\mathrm{C}-\mathrm{H} / \pi$ interactions between the two.

In the dearomative transition states, the ketone-based Me group occupies an axial position and is placed in a quadrant occupied by either a ${ }^{t} \mathrm{Bu}$ group on L1 (TS-3) or a Ph group on L4 (TS-3'). The substrate-ligand steric repulsion with the ${ }^{t} \mathrm{Bu}$ group on $\mathbf{L 1}$ destabilizes $\mathbf{T S}-\mathbf{3}$, which features a short $\mathrm{H} \cdots \mathrm{H}$ distance of $1.96 \AA$; whereas the L4-supported TS-3' experiences diminished substrate-ligand steric repulsion along with stabilizing $\mathrm{C}-\mathrm{H} / \pi$ interaction between the olefin-based $\mathrm{Me}$ group and $\mathrm{Ph}$ group on the ligand (Figure 3B). In the exocyclic addition transition states (TS2 and TS-2'), the axial ketone-based Me group occupies a less crowded quadrant, and thus the most significant interactions arise between the heterocycle and ligand. In the L1-supported TS-2, the distance between the ligandbased ${ }^{t} \mathrm{Bu} \mathrm{C}-\mathrm{H}$ bond and the indole $\pi$-system is $2.59 \AA$, indicating a stabilizing $\mathrm{C}-\mathrm{H} / \pi$ interaction. By contrast, $\mathrm{C}-$ $H / \pi$ or $\pi / \pi$ interaction was not observed in TS-2', where the $\mathrm{Ph}$ group on $\mathbf{L 4}$ is relatively far away from the heteroaryl substrate (Figure 3B).

In the most favorable exocyclic and dearomative ketone addition transition states (TS-2 and TS-3' with L1 and L4, respectively), the larger $\mathrm{Ph}$ group on the ketone prefers the equatorial position to avoid repulsions with the heteroaryl groups $^{76}$ (see Figure 3C for higher energy transition states with Ph group at the axial position). This orientation of ketone within the six-membered cyclic transition states determines the diastereoselectivity of the reaction.

The origin of the selective generation of $Z$-dearomatized products is due to the preferred orientation of the olefinbased Me group at the axial position in the six-membered cyclic transition state (TS-3'). An equatorial arrangement (TS-5') forces the Me group into an unfavorable eclipsed conformation against the heteroarene (Figure 3C), diminishing the accessibility to the $E$-stereoisomer of the product.

In summary, we have developed a protocol for the selective reductive coupling between vinyl heteroarenes and ketones to rapidly construct enantioenriched tertiary 
alcohols with adjacent stereocenters. The transformation exhibits regiodivergent reactivity controlled by the ancillary ligand employed, yielding either the exocyclic or dearomatized addition product. This method is tolerant of a wide range of important functional groups prevalent in pharmaceuticals and biologically active natural products. The mechanism through which the chiral catalyst exerts enantio- and diastereocontrol was elucidated by theoretical investigations.

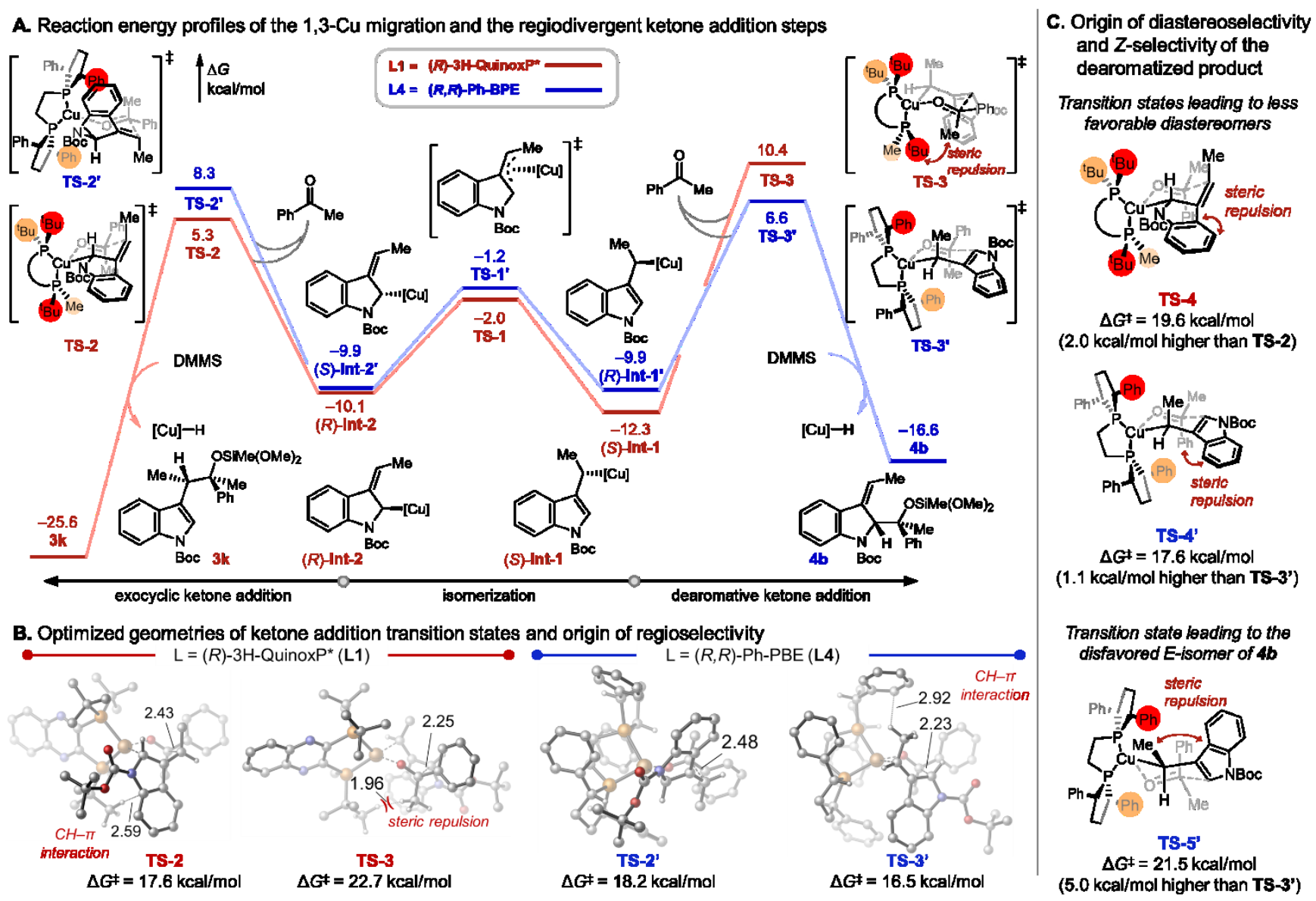

Figure 3. Computational studies of the mechanism and selectivity of the regiodivergent reductive coupling between $\mathbf{5}$ and $\mathbf{2 a}$ using $(R)$-3H-QuinoxP* (L1, red) and $(R, R)$-Ph-BPE $(\mathbf{L} 4$, blue). Bond distances are in $\AA$. Gibbs free energies are with respect to LCuH and the starting materials in (A) and with respect to the alkyl-Cu species $(S)$-Int-1 and $(R)$-Int-1' in (B) and (C).

\section{ASSOCIATED CONTENT}

Supporting Information.

The Supporting Information is available free of charge via the Internet at http://pubs.acs.org.

Experimental procedures and characterization data for all new compounds, including NMR spectra, SFC, and X-ray crystallographic information, computational details, and Cartesian coordinates of all computed structures (PDF)

\section{AUTHOR INFORMATION}

\section{Corresponding Authors}

Peng Liu - Department of Chemistry, University of Pittsburgh, Pittsburgh, Pennsylvania 15260, United States; orcid.org/0000-0002-8188-632X; Email: pengliu@pitt.edu

Stephen L. Buchwald - Department of Chemistry, Massachusetts Institute of Technology, Cambridge, Massachusetts 02139, United States; orcid.org/0000-00033875-4775; Email: sbuchwal@mit.edu

\section{Authors}

Yuyang Dong - Department of Chemistry, Massachusetts Institute of Technology, Cambridge, Massachusetts 02139, United States; orcid.org/0000-0002-4533-4798

Alexander W. Schuppe - Department of Chemistry, Massachusetts Institute of Technology, Cambridge, Massachusetts 02139, United States; orcid.org/0000- 00016002-9110

Binh Khanh Mai - Department of Chemistry, University of Pittsburgh, Pittsburgh, Pennsylvania 15260, United States; orcid.org/0000-0001-8487-1417

Notes

The authors declare no competing financial interest

\section{ACKNOWLEDGMENT}

Research reported in this publication was supported by the Arnold and Mabel Beckman Foundation for a postdoctoral fellowship to A.W.S., and the National Institutes of Health (R35GM122483). We thank the National Institutes of Health for a supplemental grant for the purchase of supercritical fluid chromatography (SFC) equipment (GM058160-17S1). We thank Nippon Chemical Industrial Co., Ltd. for the generous donation of $(R, R)$-QuinoxP*, $(R, R)$-BenzP, $(R)$-3H-QuinoxP, and $(R, R)$-BiphenylP ligands. We thank Prof. Hajime Ito for the generous donation of $(R, R)-5,8$-TMS-QuinoxP* ligand. P.L and 
B.K.M acknowledge the University of Pittsburgh and the NIH (R35GM128779) for financial support. DFT calculations were carried out at the Center for Research Computing at the University of Pittsburgh, the Extreme Science and Engineering Discovery Environment (XSEDE) supported by the National Science Foundation grant number ACI-1548562. We are grateful to, Drs. Simon Rössler, Hojoon Park, and Christine Nguyen (MIT) for advice on the preparation of this manuscript.

\section{REFERENCES}

1. He, Z.-T.; Jiang, X.; Hartwig, J. F., Stereodivergent Construction of Tertiary Fluorides in Vicinal Stereogenic Pairs by Allylic Substitution with Iridium and Copper Catalysts. J. Am. Chem. Soc. 2019, 141, 13066-13073.

2. Jiang, X.; Boehm, P.; Hartwig, J. F., Stereodivergent Allylation of Azaaryl Acetamides and Acetates by Synergistic Iridium and Copper Catalysis. J. Am. Chem. Soc. 2018, 140, 1239-1242.

3. Li, C.; Liu, R. Y.; Jesikiewicz, L. T.; Yang, Y.; Liu, P.; Buchwald, S. L., CuH-Catalyzed Enantioselective Ketone Allylation with 1,3-Dienes: Scope, Mechanism, and Applications. J. Am. Chem. Soc. 2019, 141, 5062-5070.

4. Li, C.; Shin, K.; Liu, R. Y.; Buchwald, S. L., Engaging Aldehydes in CuH-Catalyzed Reductive Coupling Reactions: Stereoselective Allylation with Unactivated 1,3-Diene Pronucleophiles. Angew. Chem. Int. Ed. 2019, 58, 17074-17080.

5. Tsai, E. Y.; Liu, R. Y.; Yang, Y.; Buchwald, S. L., A Regio- and Enantioselective CuH-Catalyzed Ketone Allylation with Terminal Allenes. J. Am. Chem. Soc. 2018, 140, 2007-2011.

6. Lee, A.; Betori, R. C.; Crane, E. A.; Scheidt, K. A., An Enantioselective Cross-Dehydrogenative Coupling Catalysis Approach to Substituted Tetrahydropyrans. J. Am. Chem. Soc. 2018, 140, 6212-6216.

$7 . \quad$ Nguyen, K. D.; Park, B. Y.; Luong, T.; Sato, H.; Garza, V. J.; Krische, M. J., Metal-catalyzed reductive coupling of olefin-derived nucleophiles: Reinventing carbonyl addition. Science 2016, 354, 5133.

8. Santana, C. G.; Krische, M. J., From Hydrogenation to Transfer Hydrogenation to Hydrogen Auto-Transfer in Enantioselective MetalCatalyzed Carbonyl Reductive Coupling: Past, Present, and Future. ACS Catal. 2021, 11, 5572-5585.

9. Xiang, M.; Pfaffinger, D. E.; Ortiz, E.; Brito, G. A.; Krische, M. J., Enantioselective Ruthenium-BINAP-Catalyzed Carbonyl Reductive Coupling of Alkoxyallenes: Convergent Construction of syn-sec,tert-Diols via (Z)- $\sigma$-Allylmetal Intermediates. J. Am. Chem. Soc. 2021, 143, 88498854.

10. Yang, Y.; Perry, I. B.; Buchwald, S. L., Copper-Catalyzed Enantioselective Addition of Styrene-Derived Nucleophiles to Imines Enabled by Ligand-Controlled Chemoselective Hydrocupration. J. Am. Chem. Soc 2016, 138, 9787-9790.

11. Agapiou, K.; Cauble, D. F.; Krische, M. J., Copper-Catalyzed Tandem Conjugate Addition-Electrophilic Trapping: Ketones, Esters, and Nitriles as Terminal Electrophiles. J. Am. Chem. Soc. 2004, 126, 45284529.

12. Yang, Y.; Perry, I. B.; Lu, G.; Liu, P.; Buchwald, S. L., Coppercatalyzed asymmetric addition of olefin-derived nucleophiles to ketones. Science 2016, 353, 144-150.

13. Klein, C.; Hüttel, W., Tertiary alcohol preferred: Hydroxylation of trans-3-methyl-L-proline with proline hydroxylases. Beilstein J. Org. Chem. 2011, 7, 1643-1647.

14. Chen, S.-T.; Fang, J.-M., Preparation of Optically Active Tertiary Alcohols by Enzymatic Methods. Application to the Synthesis of Drugs and Natural Products. J. Org. Chem. 1997, 62, 4349-4357.

15. Palomo, C.; Oiarbide, M.; García, J. M., The Aldol Addition Reaction: An Old Transformation at Constant Rebirth. Chem. Eur. J. 2002, 8, 36-44.

16. Shibasaki, M.; Kanai, M., Asymmetric Synthesis of Tertiary Alcohols and $\alpha$-Tertiary Amines via Cu-Catalyzed $\mathrm{C}-\mathrm{C}$ Bond Formation to Ketones and Ketimines. Chem. Rev. 2008, 108, 2853-2873.

17. Riant, O.; Hannedouche, J., Asymmetric catalysis for the construction of quaternary carbon centres: nucleophilic addition on ketones and ketimines. Org. Biomol. Chem. 2007, 5, 873-888.

$18 . \quad J o h n s o n$, J. S.; Evans, D. A., Chiral Bis(oxazoline) Copper(II) Complexes: Versatile Catalysts for Enantioselective Cycloaddition, Aldol, Michael, and Carbonyl Ene Reactions. Acc. Chem. Res. 2000, 33, 325-335. 19. Beletskaya, I. P.; Nájera, C.; Yus, M., Stereodivergent Catalysis. Chem. Rev. 2018, 118, 5080-5200.
20. Osakama, K.; Nakajima, M., Asymmetric Direct 1,2-Addition of Aryl Grignard Reagents to Aryl Alkyl Ketones. Org. Lett. 2016, 18, 236239.

21. Collados, J. F.; Solà, R.; Harutyunyan, S. R.; Maciá, B., Catalytic Synthesis of Enantiopure Chiral Alcohols via Addition of Grignard Reagents to Carbonyl Compounds. ACS Catal. 2016, 6, 19521970 .

22. Trost, B. M.; Weiss, A. H., The Enantioselective Addition of Alkyne Nucleophiles to Carbonyl Groups. Adv. Synth. Catal. 2009, 351, 963-983.

23. Tracy, J. S.; Kalnmals, C. A.; Toste, F. D., Beyond Allylic Alkylation: Applications of Trost Chemistry in Complex Molecule Synthesis. Isr. J. Chem. 2021, 61, 340-366.

24. Liu, Y.-L.; Lin, X.-T., Recent Advances in Catalytic Asymmetric Synthesis of Tertiary Alcohols via Nucleophilic Addition to Ketones. Adv. Synth. Catal. 2019, 361, 876-918.

25. Luderer, M. R.; Bailey, W. F.; Luderer, M. R.; Fair, J. D.; Dancer, R. J.; Sommer, M. B., Asymmetric addition of achiral organomagnesium reagents or organolithiums to achiral aldehydes or ketones: a review. Tetrahedron: Asymmetry 2009, 20, 981-998.

26. Xiang, M.; Pfaffinger, D. E.; Krische, M. J., Allenes and Dienes as Chiral Allylmetal Pronucleophiles in Catalytic Enantioselective $\mathrm{C}=\mathrm{X}$ Addition: Historical Perspective and State-of-The-Art Survey. Chem. Eur. J. 2021, 27, 13107-13116.

27. Holmes, M.; Schwartz, L. A.; Krische, M. J., Intermolecular Metal-Catalyzed Reductive Coupling of Dienes, Allenes, and Enynes with Carbonyl Compounds and Imines. Chem. Rev. 2018, 118, 6026-6052.

28. Doerksen, R. S.; Meyer, C. C.; Krische, M. J., Feedstock Reagents in Metal-Catalyzed Carbonyl Reductive Coupling: Minimizing Preactivation for Efficiency in Target-Oriented Synthesis. Angew. Chem. Int. Ed. 2019, 58, 14055-14064.

$29 . \quad$ Cooze, C.; Dada, R.; Lundgren, R. J., Direct Formic Acid Mediated Z-Selective Reductive Coupling of Dienes and Aldehydes. Angew. Chem. Int. Ed. 2019, 58, 12246-12251.

30. Wang, H.; Dai, X.-J.; Li, C.-J., Aldehydes as alkyl carbanion equivalents for additions to carbonyl compounds. Nat. Chem. 2017, 9, 374378.

31. Xiang, M.; Ghosh, A.; Krische, M. J., Diastereo- and Enantioselective Ruthenium-Catalyzed C-C Coupling of 1-Arylpropynes and Alcohols: Alkynes as Chiral Allylmetal Precursors in Carbonyl anti- $(\alpha-$ Aryl)allylation. J. Am. Chem. Soc. 2021, 143, 2838-2845.

32. Meyer, C. C.; Ortiz, E.; Krische, M. J., Catalytic Reductive Aldol and Mannich Reactions of Enone, Acrylate, and Vinyl Heteroaromatic Pronucleophiles. Chem. Rev. 2020, 120, 3721-3748.

33. Spielmann, K.; Xiang, M.; Schwartz, L. A.; Krische, M. J., Direct Conversion of Primary Alcohols to 1,2-Amino Alcohols: Enantioselective Iridium-Catalyzed Carbonyl Reductive Coupling of Phthalimido-Allene via Hydrogen Auto-Transfer. J. Am. Chem. Soc. 2019, 141, 14136-14141.

34. Pirnot, M. T.; Wang, Y.-M.; Buchwald, S. L., Copper Hydride Catalyzed Hydroamination of Alkenes and Alkynes. Angew. Chem. Int. Ed. 2016, 55, 48-57.

35. Liu, R. Y.; Buchwald, S. L., CuH-Catalyzed Olefin Functionalization: From Hydroamination to Carbonyl Addition. Acc. Chem. Res. 2020, 53, 1229-1243.

36. Jang, W. J.; Yun, J., Copper-Catalyzed Tandem Hydrocupration and Diastereo- and Enantioselective Borylalkyl Addition to Aldehydes. Angew. Chem. Int. Ed. 2018, 57, 12116-12120.

37. Chuzel, O.; Deschamp, J.; Chausteur, C.; Riant, O., Copper(I)Catalyzed Enantio- and Diastereoselective Tandem Reductive Aldol Reaction. Org. Lett. 2006, 8, 5943-5946.

38. Dora, C. S., Additions to non-activated alkenes: Recent advances. Arab. J. Chem. 2020, 13, 799-834.

39. Liu, R. Y.; Zhou, Y.; Yang, Y.; Buchwald, S. L., Enantioselective Allylation Using Allene, a Petroleum Cracking Byproduct. J. Am. Chem. Soc. 2019, 141, 2251-2256.

40. Saxena, A.; Choi, B.; Lam, H. W., Enantioselective CopperCatalyzed Reductive Coupling of Alkenylazaarenes with Ketones. J. Am. Chem. Soc. 2012, 134, 8428-8431.

41. Trost, B. M.; Czabaniuk, L. C., Structure and Reactivity of Late Transition Metal $\eta 3$-Benzyl Complexes. Angew. Chem. Int. Ed. 2014, 53, 2826-2851.

42. Wertjes, W. C.; Southgate, E. H.; Sarlah, D., Recent advances in chemical dearomatization of nonactivated arenes. Chem. Soc. Rev. 2018, 47, 7996-8017.

43. Lipshutz, B. H.; Chrisman, W.; Noson, K., Hydrosilylation of aldehydes and ketones catalyzed by $\left[\mathrm{Ph}_{3} \mathrm{P}(\mathrm{CuH})\right]_{6}$. J. Organomet. Chem. 2001, 624, 367-371. 
44. Moser, R.; Bošković, Ž. V.; Crowe, C. S.; Lipshutz, B. H., CuHCatalyzed Enantioselective 1,2-Reductions of $\alpha, \beta$-Unsaturated Ketones. $J$. Am. Chem. Soc 2010, 132, 7852-7853.

45. Chen, J.-X.; Daeuble, J. F.; Brestensky, D. M.; Stryker, J. M., Highly Chemoselective Catalytic Hydrogenation of Unsaturated Ketones and Aldehydes to Unsaturated Alcohols Using Phosphine-Stabilized Copper(I) Hydride Complexes. Tetrahedron 2000, 56, 2153-2166.

46. Liu, R. Y.; Yang, Y.; Buchwald, S. L., Regiodivergent and Diastereoselective $\mathrm{CuH}$-Catalyzed Allylation of Imines with Terminal Allenes. Angew. Chem. Int. Ed. 2016, 55, 14077-14080.

47. Gribble, M. W.; Liu, R. Y.; Buchwald, S. L., Evidence for Simultaneous Dearomatization of Two Aromatic Rings under Mild Conditions in $\mathrm{Cu}(\mathrm{I})$-Catalyzed Direct Asymmetric Dearomatization of Pyridine. J. Am. Chem. Soc. 2020, 142, 11252-11269.

48. Tian, Q.; Bai, J.; Chen, B.; Zhang, G., Chromium-Catalyzed Asymmetric Dearomatization Addition Reactions of Halomethyl Heteroarenes. Org. Lett. 2016, 18, 1828-1831.

49. Bao, M.; Nakamura, H.; Yamamoto, Y., Facile Allylative Dearomatization Catalyzed by Palladium. J. Am. Chem. Soc. 2001, 123, 759-760.

50. $\quad$ Peng, B.; Zhang, S.; Yu, X.; Feng, X.; Bao, M., Nucleophilic Dearomatization of Chloromethyl Naphthalene Derivatives via $\eta^{3}$ Benzylpalladium Intermediates: A New Strategy for Catalytic Dearomatization. Org. Lett. 2011, 13, 5402-5405.

51. Zhang, S.; Yu, X.; Feng, X.; Yamamoto, Y.; Bao, M., Palladium-catalyzed regioselective allylation of five-membered heteroarenes with allyltributylstannane. Chem. Commun. 2015, 51, 38423845 .

52. Wu, W.-T.; Zhang, L.; You, S.-L., Catalytic asymmetric dearomatization (CADA) reactions of phenol and aniline derivatives. Chem. Soc. Rev. 2016, 45, 1570-1580.

53. Zhuo, C.-X.; Zheng, C.; You, S.-L., Transition-Metal-Catalyzed Asymmetric Allylic Dearomatization Reactions. Acc. Chem. Res. 2014, 47, 2558-2573.

54. Sharma, U. K.; Ranjan, P.; Van der Eycken, E. V.; You, S.-L., Sequential and direct multicomponent reaction (MCR)-based dearomatization strategies. Chem. Soc. Rev. 2020, 49, 8721-8748.

55. Zheng, C.; You, S.-L., Advances in Catalytic Asymmetric Dearomatization. ACS Cent. Sci. 2021, 7, 432-444.

56. You, S.-L., Asymmetric Dearomatization Reactions. Wiley VCH: 2016

57. Zheng, C.; You, S.-L., Catalytic Asymmetric Dearomatization by Transition-Metal Catalysis: A Method for Transformations of Aromatic Compounds. Chem 2016, 1, 830-857.

58. Gribble, M. W.; Guo, S.; Buchwald, S. L., Asymmetric CuCatalyzed 1,4-Dearomatization of Pyridines and Pyridazines withou Preactivation of the Heterocycle or Nucleophile. J. Am. Chem. Soc. 2018 140, 5057-5060.

59. McAtee, R. C.; Noten, E. A.; Stephenson, C. R. J., Arene dearomatization through a catalytic $\mathrm{N}$-centered radical cascade reaction. Nat. Commun. 2020, 11, 2528.

60. Hudlicky, T.; Reed, J. W., Celebrating 20 Years of SYNLETT Special Account On the Merits of Biocatalysis and the Impact of Arene cisDihydrodiols on Enantioselective Synthesis. Synlett 2009, 2009, 685-703.

61. Li, X.; Duan, M.; Yu, P.; Houk, K. N.; Sun, J., Organocatalytic enantioselective dearomatization of thiophenes by 1,10 -conjugate addition of indole imine methides. Nat. Commun. 2021, 12, 4881.
62. Huang, X.; Zhang, Y.; Liang, W.; Zhang, Q.; Zhan, Y.; Kong, L.; Peng, B., Dearomatization of aryl sulfoxides: a switch between monoand dual-difluoroalkylation. Chem. Sci. 2020, 11, 3048-3053.

63. Roche, S. P.; Porco Jr., J. A., Dearomatization Strategies in the Synthesis of Complex Natural Products. Angew. Chem. Int. Ed. 2011, 50, 4068-4093.

64. Nair, V. N.; Kojasoy, V.; Laconsay, C. J.; Kong, W. Y.; Tantillo, D. J.; Tambar, U. K., Catalyst-Controlled Regiodivergence in Rearrangements of Indole-Based Onium Ylides. J. Am. Chem. Soc. 2021 143, 9016-9025.

65. Trammel, G. L.; Kuniyil, R.; Crook, P. F.; Liu, P.; Brown, M. K., Nickel-Catalyzed Dearomative Arylboration of Indoles: Regioselective Synthesis of C2- and C3-Borylated Indolines. J. Am. Chem. Soc. 2021, 143, 16502-16511.

66. $\mathrm{Hu}, \mathrm{Q}$.; Chen, J.; Zhang, Z.; Liu, Y.; Zhang, W., Rh-Catalyzed One-Pot Sequential Asymmetric Hydrogenation of $\alpha$-Dehydroamino Ketones for the Synthesis of Chiral Cyclic trans- $\beta$-Amino Alcohols. Org. Lett. 2016, 18, 1290-1293.

67. Iwamoto, H.; Imamoto, T.; Ito, H., Computational design of high-performance ligand for enantioselective Markovnikov hydroboration of aliphatic terminal alkenes. Nat. Commun. 2018, 9, 2290.

68. Jia, J.; Ling, Z.; Zhang, Z.; Tamura, K.; Gridnev, I. D.; Imamoto, T.; Zhang, W., An Atropos Chiral Biphenyl Bisphosphine Ligand Bearing Only 2,2' -Substituents and Its Application in Rh-Catalyzed Asymmetric Hydrogenation. Adv. Synth. Catal. 2018, 360, 738-743.

69. Iwamoto, H.; Ozawa, Y.; Takenouchi, Y.; Imamoto, T.; Ito, H., Backbone-Modified $C_{2}$-Symmetrical Chiral Bisphosphine TMS-QuinoxP* Asymmetric Borylation of Racemic Allyl Electrophiles. J. Am. Chem. Soc. 2021, 143, 6413-6422.

70. Heravi, M. M.; Zadsirjan, V., Prescribed drugs containing nitrogen heterocycles: an overview. RSC Adv. 2020, 10, 44247-44311.

71. Zhang, T. Y., Chapter One - The Evolving Landscape of Heterocycles in Drugs and Drug Candidates. In Adv. Heterocycl. Chem., Scriven, E. F. V.; Ramsden, C. A., Eds. Academic Press: 2017; Vol. 121, pp 1-12.

72. Vitaku, E.; Smith, D. T.; Njardarson, J. T., Analysis of the Structural Diversity, Substitution Patterns, and Frequency of Nitrogen Heterocycles among U.S. FDA Approved Pharmaceuticals. J. Med. Chem. 2014, 57, 10257-10274.

73. Baker, B. A.; Bošković, Ž. V.; Lipshutz, B. H., (BDP)CuH: A "Hot" Stryker's Reagent for Use in Achiral Conjugate Reductions. Org. Lett. 2008, 10, 289-292.

74. Appella, D. H.; Moritani, Y.; Shintani, R.; Ferreira, E. M.; Buchwald, S. L., Asymmetric Conjugate Reduction of $\alpha, \beta$-Unsaturated Esters Using a Chiral Phosphine-Copper Catalyst. J. Am. Chem. Soc. 1999, 121, 9473-9474

75. Mahoney, W. S.; Brestensky, D. M.; Stryker, J. M., Selective hydride-mediated conjugate reduction of $\alpha, \beta$-unsaturated carbonyl compounds using $\left[\left(\mathrm{Ph}_{3} \mathrm{P}\right) \mathrm{CuH}\right]_{6}$. J. Am. Chem. Soc. 1988, 110, 291-293. 76. Ye, Y.; Kevlishvili, I.; Feng, S.; Liu, P.; Buchwald, S. L., Highly Enantioselective Synthesis of Indazoles with a C3-Quaternary Chiral Center Using CuH Catalysis. J. Am. Chem. Soc. 2020, 142, 10550-10556. 


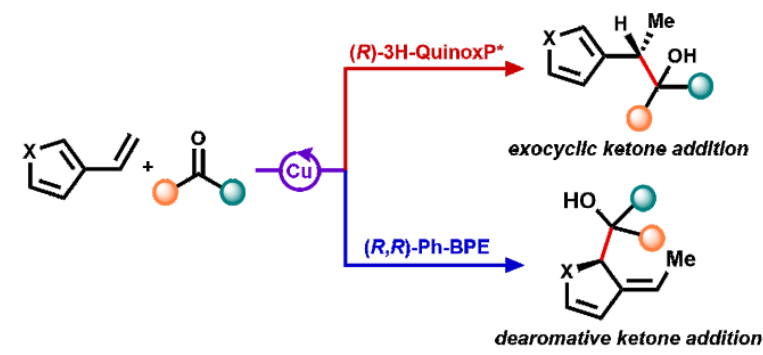

8 